\title{
BMJ Open Endobronchial ultrasound in diagnosing and staging of lung cancer by Acquire 22G TBNB versus regular 22G TBNA needles: study protocol of a randomised clinical trial
}

\author{
Jolanda C Kuijvenhoven, ${ }^{1,2}$ Tess Kramer (D) , ${ }^{1}$ Daniël A Korevaar, ${ }^{1}$ \\ Maarten K Ninaber, ${ }^{3}$ Rocco Trisolini, ${ }^{4}$ Artur Szlubowski, ${ }^{5}$ Maciej Gnass, ${ }^{5}$ \\ Jan von der Thüsen (1) , ${ }^{6}$ Danielle Cohen, ${ }^{7}$ Peter I Bonta, ${ }^{1}$ Jouke T Annema ${ }^{1}$
}

To cite: Kuijvenhoven JC, Kramer T, Korevaar DA, et al. Endobronchial ultrasound in diagnosing and staging of lung cancer by Acquire 22G TBNB versus regular 22G TBNA needles: study protocol of a randomised clinical trial. BMJ Open 2021;11:e051820. doi:10.1136/ bmjopen-2021-051820

- Prepublication history for this paper is available online. To view these files, please visit the journal online (http://dx.doi org/10.1136/bmjopen-2021051820).

JCK and TK are joint first authors.

Received 30 March 2021 Accepted 13 August 2021

Check for updates

(C) Author(s) (or their employer(s)) 2021. Re-use permitted under CC BY-NC. No commercial re-use. See rights and permissions. Published by BMJ.

For numbered affiliations see end of article.

Correspondence to

Professor Jouke T Annema;

j.t.annema@amsterdamumc.nl

\section{ABSTRACT}

Introduction Accurate diagnosis and staging of lung cancer is crucial because it directs treatment and prognosis. Endobronchial ultrasound-guided transbronchial needle aspiration (EBUS-TBNA) and endoscopic ultrasound with bronchoscope fine-needle aspiration (EUS-B-FNA) are important in this process by sampling hilar/mediastinal lymph nodes and centrally located lung tumours. With the upcoming of immunotherapy and targeted therapies, assessment of programmed death ligand 1 (PD-L1) expression and molecular profiling has become important but is often impossible in cytological samples obtained through standard 22G TBNA needles. Recently, a threepronged cutting edge $22 \mathrm{G}$ needle was developed that allows for transbronchial needle biopsy (TBNB). Our objective is to determine if EBUS/EUS-B-guided nodal/lung tumour sampling with Acquire 22G TBNB needles results in an improved suitability rate for the assessment of PD-L1 expression in comparison to standard 22G TBNA needles in patients with a final diagnosis of lung cancer.

Methods and analysis This is an investigator-initiated, parallel group randomised clinical trial. Patients are recruited at respiratory medicine outpatient clinics of participating university and general hospitals in the Netherlands, Poland and Italy. In total 158 adult patients with (suspected) lung cancer are included if they have an indication for mediastinal/hilar lymph node or lung tumour sampling by EBUS-TBNA and/or EUS-B-FNA based on current clinical guidelines. Web-based randomisation between the two needles will be performed. Samples obtained from mediastinal/hilar lymph nodes and/or primary tumour will be processed for cytology smears and cell block analysis and reviewed by blinded reference pathologists. An intentionto-treat analysis will be applied. Patients with missing data will be excluded from analysis for that specific variable but included in the analysis of other variables. This study is financially supported by Boston Scientific.

Ethics and dissemination The study was approved by the local Ethics Committee (Medisch Ethische Toetsingscommissie Amsterdam Medical Center (AMC)). Dissemination will involve publication in a peer-reviewed biomedical journal.

\section{Strengths and limitations of this study}

- The international, multicentre design will improve generalisability of the findings.

- Two blinded reference pathologists will analyse the tissue samples in order to objectively compare the two needles.

- The Acquire 22G transbronchial needle biopsy (TBNB) needle is commercially available and therefore, our findings can directly be implemented in clinical practice.

- Performing bronchoscopists cannot be blinded to needle randomisation which is a potential source of performance bias.

- This study is not powered to assess if the Acquire 22G TBNB results in an improved diagnostic yield compared with the $22 \mathrm{G}$ transbronchial needle aspiration.

Trial registration number NL7701; Pre-results.

\section{INTRODUCTION}

Lung cancer is the most commonly diagnosed cancer worldwide with the highest mortality of all cancers. ${ }^{1}$ Accurate staging is important because it directs treatment and prognosis. $^{2}{ }^{3}$ Mediastinal and hilar staging is key in this process. For decades, this was done by (cervical) mediastinoscopy which is a costly and invasive procedure with suboptimal sensitivity for mediastinal metastases. ${ }^{4}$ Over the past 15 years, clinicians have started to use endobronchial ultrasound-guided transbronchial needle aspiration (EBUSTBNA) for this purpose which is less invasive and has a similar diagnostic range to cervical mediastinoscopy. ${ }^{5}$ Bronchial EBUS-TBNA can be combined with endoscopic ultrasound fine-needle aspiration (EUS-FNA) from the 
oesophagus, which can reach additional lymph node stations located in the lower and posterior mediastinum. ${ }^{6}$ It has been shown that a combined endosonography investigation (EBUS and EUS), followed by mediastinoscopy in the absence of metastases at endosonography, results in greater sensitivity for mediastinal nodal metastases compared with mediastinoscopy alone. ${ }^{5}$ Therefore, current guidelines on lung cancer staging recommend endosonography (EBUS and/or EUS) as the initial tissue sampling technique for mediastinal nodal staging. ${ }^{2}$ Additionally, EBUS and EUS can be used to sample centrally located lung tumours. ${ }^{7-9}$

Over the past years, the treatment options for patients with advanced lung cancer have been expanding rapidly. Encouragingly, targeted immunotherapies against programmed death ligand 1 (PD-L1) (eg, durvalumab and atezolizumab) and its receptor PD-1 (eg, nivolumab and pembrolizumab) have improved the survival in selected patients with advanced lung cancer. Therefore, to be able to assign a patient to optimal treatment, assessment of PD-L1 expression and molecular profiling of the tumour is crucial. With the advent of EBUS-TBNA and EUS-FNA, the ability to accurately assess the PD-L1 expression and molecular profiling on small, cytological tissue samples has become of great importance. Although the use of cell blocks and improved sequencing techniques have expanded the possibilities for PD-L1 assessment, ${ }^{10-14}$ the success rate of PD-L1 expression and molecular profiling remains suboptimal at cytology compared with histology. ${ }^{15-17}$

Recently, a three-plane symmetric needle with Franseen geometry (Acquire 22G transbronchial needle biopsy $(\mathrm{TBNB})$ ) was developed (figure 1). In pancreatic cysts, the Acquire 22G needle allowed improved, true histological core tissue acquisition in pancreatic cysts. ${ }^{18} 19$ To date, there is no literature about the Acquire 22G TBNB needle for diagnostic and staging purposes during EBUS/endoscopic ultrasound with bronchoscope (EUS-B) procedures in patients with (suspected) lung cancer.

In this parallel group randomised clinical trial, we hypothesise that EBUS/EUS-B sampling of mediastinal/

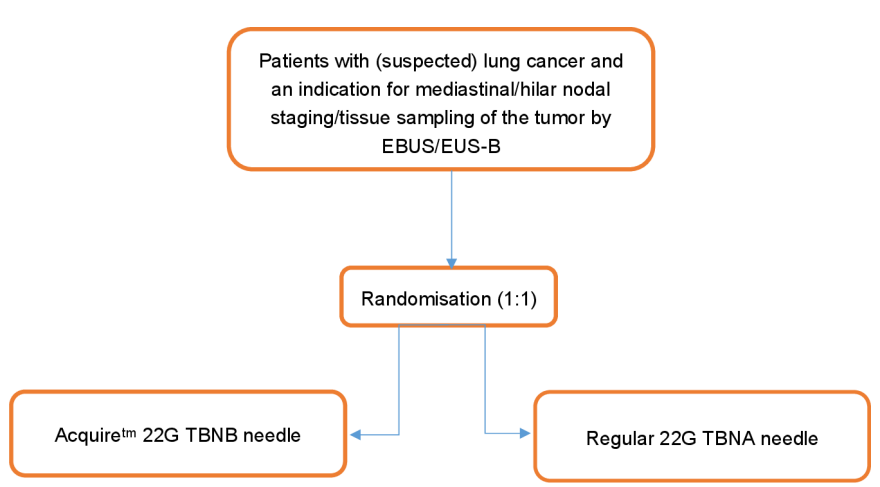

Figure 1 Flow chart of study design. EBUS, endobronchial ultrasound; EUS-B, endoscopic ultrasound with bronchoscope; TBNA, transbronchial needle aspiration; TBNB, transbronchial needle biopsy. hilar lymph nodes and/or primary lung tumours with the Acquire 22G TBNB needle has a higher suitability rate for the assessment of PD-L1 expression in comparison to the regular 22G TBNA needle in patients with lung cancer.

\section{METHODS AND ANALYSIS \\ Design and study dates}

This is a protocol of an investigator-initiated parallelgroup randomised clinical trial. The trial is executed in four centres including both university and general hospitals in The Netherlands (Amsterdam University Medical Centers and Leiden University Medical Center), Italy (Fondazione Policlinico Universitario Agostino Gemelli, Rome) and Poland (Pulmonary Hospital Zakopane). We expect that we will complete inclusion in June 2022.

\section{Study population}

Patients will be recruited at outpatient clinics of respiratory medicine of the participating hospitals by their treating physicians. If the patient is willing to participate in the study, information about the study will be provided by the local investigator, who will then gain written informed consent. Eligible are patients with proven or suspected lung cancer (either non-small-cell lung cancer or small-cell lung cancer), who have an indication for mediastinal/hilar lymph node or lung tumour sampling by EBUS-TBNA and/or EUS-B-FNA according to current clinical guidelines. ${ }^{2}$ Indications for mediastinal/hilar lymph node staging are: (1) suspicion of mediastinal or hilar lymph node metastases based either on size (short axis $>10 \mathrm{~mm}$ on CT) or increased fluorodeoxyglucose (FDG) uptake, (2) a primary tumour without FDG uptake, (3) a primary tumour with a size of $\geq 3 \mathrm{~cm}$ or (4) a centrally located primary tumour. Sampling of centrally located lung tumours is attempted if the intrapulmonary mass is located adjacent to the oesophagus or airway wall but not endobronchially visible with conventional bronchoscopy. The following exclusion criteria will be applied: (1) mediastinal restaging after neoadjuvant treatment, (2) contraindication for EBUS or EUS-B (eg, severe respiratory insufficiency), (3) a non-correctable coagulation disorder, (4) pregnancy or (5) inability to consent.

\section{Study withdrawal}

Subjects can leave the study at any time for any reason if they wish to do so. The investigator can decide to withdraw a subject from the study for unanticipated urgent medical reasons. Such withdrawals will be monitored and reported in the final study report.

\section{Randomisation and needle allocation}

Randomisation will be performed prior to endosonography. Participants will be randomised to either the Boston Scientific Acquire 22G TBNB needle or the regular Boston Scientific 22G TBNA needle (figure 2). Both needles have a Conformité Européenne (CE) mark. We will use a web-based block-randomisation 


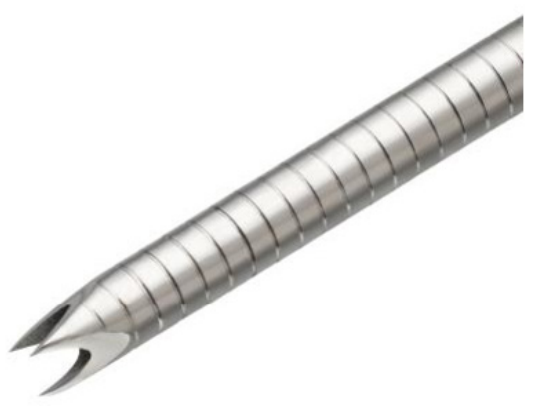

$B$

A

Figure 2 (A) Acquire 22G TBNB needle with a threepronged cutting edge, $(\mathrm{B})$ regular 22G TBNA needle. TBNA, transbronchial needle aspiration; transbronchial needle biopsy.

(using Castor Electronic Data Capture (EDC) software, Amsterdam, the Netherlands), stratified by participating hospital to ensure that both groups are of equal size for every hospital. Endoscopists will not be blinded to needle randomisation but the reference pathologists will be blinded for the procurement of the tissue.

\section{Endosonographic procedure}

Endosonography will be performed at the department of pulmonary medicine of participating hospitals by experienced chest physicians who are specifically trained in EBUS and EUS-B. Procedures will be performed according to institutional practice, mostly on an outpatient basis. Topical anaesthesia is applied to the pharynx, larynx, trachea and bronchi. Sedation will be administered according to institutional practice, mostly conscious sedation by midazolam $2.5-5 \mathrm{mg}$ intravenous with or without fentanyl or propofol sedation. Vital parameters will be monitored during and after the procedure. Patients will be observed for 1 hour after the endosonography procedure.

Procedures will be performed using an ultrasound bronchoscope with a linear scanning transducer (EBUS scope). A dedicated ultrasound processor is used for imaging processing with Doppler flow imaging for the detection of blood vessels and that enables real-time ultrasound guidance for sampling. All patients will undergo a systematic endosonographic evaluation of all the accessible mediastinal/hilar lymph nodes and the tumour itself. $^{2} 20$ Following the endosonographic inspection, lymph nodes will be sampled with the randomised needle from N3 to N2 to N1 to the tumour itself. A minimum of two needle passes will be performed for each lymph node and tumour. After insertion of the needle in the target lymph node or tumour, the stylet will be pulled out slowly (slow pull technique). In case of no or limited aspirate after one needle pass, suction using a $10 \mathrm{~mL}$ syringe during the following aspirations will be applied.

\section{Endoscopist satisfaction}

After the endoscopic procedure, the endoscopists satisfaction score (scale $0-10$, where 0 is the lowest score and 10 the highest) will be scored.

\section{Sample processing}

Handling of the aspirates will be performed according to institutional practice. Nodal aspirates will be assessed by the local pathologist for clinical decision making. Rapid on-site evaluation (ROSE) is optional. When ROSE is performed, smears of the fine-needle aspirate obtained by EBUS-TBNA or EUS-B-FNA of the intrathoracic lymph nodes are performed in situ. All aspirates (both from lymph nodes as well as the tumour) will be processed for both cytology smears and cell block analysis following local practice. The local pathologist will analyse the nodal aspirates, including assessment of PD-L1 expression and molecular analysis for treatment. In case of $\mathrm{N} 0 / \mathrm{N} 1$ disease based on the TBNB/TBNA specimens, surgical pathological staging or clinical radiological follow-up at 6 months serves as reference standard for mediastinal/hilar lymph node metastases (N0/N1 vs N2/N3 disease). Which of the two is performed will be decided by the treating physician, as part of clinical practice. In case no malignancy is detected based on TBNB/TBNA specimens, the final diagnosis after 6 months follow-up will serve as the reference standard.

\section{Independent cytopathology review}

The local pathologist will assess the sampling material, including cell blocks and cytological smears for clinical purposes. After inclusion of all patients, all cell blocks will be digitalised and reviewed by two reference pathologists for independent cytopathological review. In case less than two cell blocks of a single patient are available, cytological smears will also be included in the independent cytopathological evaluation. The reference pathologists are blinded to needle randomisation but supplied with the clinical information, endosonography route (EBUS or EUS-B), results of the other tests and are aware of the differential diagnosis prior to endoscopy.

The pathologists will evaluate each cell block for the suitability of PD-L1 expression. Cell block specimens will be considered suitable if more than 100 tumour cells are present in the specimen. Additionally, the suitability for molecular analysis will be evaluated based on the presence/absence of $>1000$ tumour cells in all the cell block material combined. Also, the cumulative length of tissue core will be measured, the sample adequacy will be evaluated based on the presence of lymphocytes or atypical cells or other pathogenic characteristics (eg, granulomas), the quality of the samples will be evaluated using a Mair's objective scoring system ${ }^{21}$ and the bloodiness of the samples will be categorised based on the percentage of blood in the microscopic field. In case the 
two independent pathologists do not fully agree on one of the above-mentioned parameters, the pathologists will conduct a consensus review of discrepant cases in order to have a clear final judgement.

\section{Outcomes}

The primary outcome is the suitability rate for the assessment of PD-L1 expression on mediastinal/hilar nodal or tumour EBUS/EUS-B aspirates in patients with a final diagnosis of lung cancer.

In the initial study design, we planned to examine the hypothesis that the use of the Acquire 22G TBNB would result in a higher rate of core tissue samples, as was reported in previous studies. ${ }^{18} 19$ However, this endpoint would primarily assess the specimen quality but would not investigate the clinical impact of improved specimen quality. Therefore, it was decided to define a primary endpoint which would comprise both specimen quality as the clinical impact. For that reason, we found that the suitability rate for PD-L1 assessment would be more informative as primary endpoint. After inclusion of five patients, a protocol amendment was submitted and approved by the local medical ethical committee and changes were updated in the trial register.

The secondary outcomes are:

- Cumulative length tissue core.

- Suitability for molecular analysis/next-generation sequencing in patients with a final diagnosis of lung cancer.

- Sample adequacy (defined as the presence of lymphocytes or atypical cells or other pathogenic characteristics (eg, granulomas)).

- Sample quality using the Mair's objective scoring system.

- Sample bloodiness.

- Diagnostic sensitivity for mediastinal/hilar nodal staging (defined as the proportion of patients that have N2/N3 disease diagnosed by EBUS/EUS-B, relative to the total number of patients with a final diagnosis of $\mathrm{N} 2 / \mathrm{N} 3$ disease as determined by the reference standard).

- Diagnostic sensitivity for malignancy (defined as the proportion of patients that have malignancy diagnosed by EBUS/EUS-B, relative to the total number of patients with a final diagnosis of malignancy as determined by the reference standard).

- Yield for diagnosing malignancy in the subgroup of patients with a centrally located lung tumour (defined as the proportion of patients that have malignancy diagnosed by EBUS/EUS-B, relative to the total number of patients with a final diagnosis of malignancy).

- Complication rate (defined as any complication occurring during or directly after the endosonographic procedure or any procedure-related complication in the follow-up period).

- Procedure duration (defined as start EBUS/EUS-B scope insertion until EBUS/EUS-B scope removal).
Endoscopists satisfaction of needle use.

\section{Sample size calculation}

Based on results on the success rate of PD-L1 assessment in the Amsterdam University Medical Centres and published literature, we expect the acquisition rate of the regular 22G TBNA needle for the assessment of PD-L1 expression in patients with lung cancer is around $64 \% .{ }^{22-24}$ We expect that this will be $86 \%$ for the Acquire 22G TBNB needle. ${ }^{19}$ In total, 120 patients with lung cancer and tumour positive samples (eg, lymph node metastasis/ primary tumour sample) are needed to show, with $\mathrm{alfa}=0.05$ and power $=0.80$, that the Acquire $22 \mathrm{G}$ needle is $22 \%$ superior to the regular 22G TBNA needle. Taking into account that $80 \%$ of included patients will have a final diagnosis of lung cancer with a tumour positive aspirate and a 5\% study drop-out, 158 patients will be included. In our opinion, the expected increase in suitability rate for PDL-1 assessment (from 64 to at least $86 \%$ ) will result in a clinically relevant improvement of lung cancer diagnosis and staging.

\section{Statistical analysis}

An intention-to-treat analysis will be applied, which implies that patients will be analysed according to the needle group they will be randomised to also if endoscopy is eventually performed with another needle due to unforeseen medical decisions or circumstances. All such cases will be clearly reported in the final manuscript. Patients who are randomised but in whom no subsequent endoscopic procedure is performed, will be excluded from analysis. All patients in whom the endoscopic procedure is initiated will be included in the analysis for the primary and secondary outcomes. If bronchoscopists fail to obtain (adequate) tissue samples, this will be considered as a failure on the primary outcome (ie, failure to assess PD-L1 expression) and all relevant secondary outcomes. Because of the prospective and mostly cross-sectional nature of the study, we anticipate minimal numbers of missing data. The number of missing data for each variable will be reported in the final manuscript; patients with missing data will be excluded from analysis only for that specific variable, but be included for the analysis of other variables. Results for continuous variables will be expressed as means and SDs, or as medians with IQRs. Categorical variables will be expressed as frequencies and percentages. $\chi^{2}$ testing will be used to compare dichotomous outcomes, including the primary outcome. Continuous variables will be compared using Student's t-tests or Mann-Whitney-U tests. A two-tailed $\mathrm{p}<0.05$ will be considered statistically significant.

\section{Patient and public involvement}

Patients or the public will not be involved in the design, conduct, reporting or dissemination plans of this research.

\section{Funding}

This is an investigator-initiated study with financial and material support from Boston Scientific. 


\section{ETHICS AND DISSEMINATION}

The study will be conducted according to the principles of the Declaration of Helsinki (64th WMA General Assembly, Fortaleza, Brazil, October 2013) and in accordance with the Medical Research Involving Human Subjects Act. The study was approved by the local Ethics Committee (Medisch Ethische Toetsingscommissie AMC).

\section{Data handling}

Data collection will be performed in the participating centres. Electronic patient record forms will be provided through web-based software (Castor EDC software, Amsterdam, the Netherlands). Data collection and analysis will be monitored according to good clinical practice. Clinical monitoring will be organised in a crossover mode where CRF files will undergo a quality check. Inclusion rate will be monitored on a monthly basis. The investigators will maintain adequate records, including signed patients informed consent forms and information on adverse events. These documents need to be kept in a secured area with limited access. All records will be signed and dated by the investigators. All records are to be retained for a period of 15 years following the date the entire clinical investigation is completed, terminated or discontinued. The anonymity and confidentiality will be guaranteed and patients identification will be coded. The code will start with the abbreviation of the hospital followed by 100 with the number of inclusion (eg, AMC1001). Patients data will be centralised by the coordinating investigator and kept under strict confidentiality according the General Data Protection Regulation. The data of the patients included in the Netherlands will stay in the Dutch database.

\section{Adverse events}

EBUS for staging and diagnosing lung cancer is a routine clinical procedure that is considered safe with a low complication rate of less than $1 \% \cdot{ }^{25}$ Adverse events are defined as any undesirable experience occurring to a subject during the study, whether or not considered related to the endosonographic procedure. All adverse events reported spontaneously by the subject or observed by the endoscopist or his staff will be recorded, as well as those occurring up to 1 week after the procedure. The investigators in the participating centres will report all serious adverse events to the coordinating investigator and principal investigator. They will report the serious adverse event to the sponsor without undue delay after obtaining knowledge of the events, as well as to the accredited medical ethics committee that approved the protocol. Only the serious adverse events with a causal association with study participation will be reported in the final study report following study completion.

\section{Annual progress report}

The sponsor/investigator will submit a summary of the progress of the trial to the accredited medical ethics committee once a year. Information will be provided on the date of inclusion of the first subject, numbers of subjects included and numbers of subjects that have completed the trial, serious adverse events/ serious adverse reactions, other problems and amendments.

\section{Dissemination}

We anticipate to report our findings in an article that will be submitted to a peer-reviewed biomedical journal.

\section{DISCUSSION}

In this multicentre, randomised clinical trial, we aim to assess for the first time whether the Acquire 22G TBNB needle results in a higher success rate of PD-L1 assessment compared with the standard 22G TBNA needle. ${ }^{19}$ This is of great importance as the current success rate of PD-L1 and molecular profiling on cytological samples remains limited compared with histology. ${ }^{15-17}$ As all ancillary tests (both immunohistochemistry as molecular testing) have to be performed on small cytological specimens, sample exhaustion frequently occurs because of insufficient tumour cells to do all tests, ${ }^{24}$ resulting in unnecessary repeat procedures and treatment delay.

The novel Acquire 22G TBNB has demonstrated a significant improved diagnostic adequacy with increased tissue acquisition in sixty patients with pancreatic masses. ${ }^{19}$ Therefore, we anticipate that the Acquire 22G TBNB during EBUS/EUS-B procedures in patients with suspected lung cancer will result in a much higher success rate of assessment of PDL-1 expression as compared with traditional needles.

In order to objectively compare the tissue specimens obtained with the Acquire 22G TBNB needle and TBNA Expect needle, two blinded reference pathologists will analyse the tissue specimens on tissue quality and suitability for PD-L1 assessment and molecular profiling. Combined with the international, multicentre design this will improve the generalisability of our findings. Although the reference pathologist will be masked to needle randomisation, it is not possible to mask the attending endoscopist, which is a potential source of performance bias. After a 6-month follow-up period, the diagnostic sensitivity for malignancy and N2/N3 staging for each needle will be calculated. However, the sample size of this study is not powered to assess whether the use of the Acquire 22G TBNB will actually result in an improved diagnostic sensitivity.

The findings of this study will provide novel insights regarding EBUS/EUS-B-guided tissue acquisition in patients with suspected lung cancer using the Acquire 22G TBNB needle, which could be a significant advantage in terms of treatment selection. As the Acquire 22G TBNB needle is commercially available, study results can directly be implemented in clinical practice.

\section{Author affiliations}

${ }^{1}$ Department of Respiratory Medicine, Amsterdam University Medical Centres, Amsterdam, The Netherlands 
${ }^{2}$ Department of Respiratory Medicine, Medical Centre Leeuwarden, Leeuwarden, The Netherlands

${ }^{3}$ Department of Respiratory Medicine, Leiden University Medical Center, Leiden, The Netherlands

${ }^{4}$ Department of Respiratory Medicine, Fondazione Policlinico Universitario Agostino Gemelli IRCCS, Roma, Italy

${ }^{5}$ Department of Respiratory Medicine, Pulmonary Hospital, Zakopane, Poland

${ }^{6}$ Department of Pathology, Erasmus MC, Rotterdam, The Netherlands

${ }^{7}$ Department of Pathology, Leiden University Medical Center, Leiden, The Netherlands

Contributors JCK, TK, DAK, MKN, JvdT, DC, PIB and JTA were involved in conception and trial design. JCK, TK, DAK and JTA were involved in drafting of the study protocol. DAK provided statistical expertise. All authors were involved in final approval of the protocol. TK, MKN, RT, AS, MG, JTA and PIB will be involved in the conduct of the study and data acquisistion. JCK, TK, JvdT and DC will be involved in the data analysis and interpretation. JCK, TK, DAK, PIB and JTA will be involved in drafting of the final manuscript for dissemeniation. All authors will provide approval of the final manuscript for publication.

Funding This work was supported by Boston Scientific.

Competing interests JTA reports finanical support from Boston Scientific

Patient and public involvement Patients and/or the public were not involved in the design, or conduct, or reporting, or dissemination plans of this research.

Patient consent for publication Not required.

Provenance and peer review Not commissioned; externally peer reviewed.

Open access This is an open access article distributed in accordance with the Creative Commons Attribution Non Commercial (CC BY-NC 4.0) license, which permits others to distribute, remix, adapt, build upon this work non-commercially, and license their derivative works on different terms, provided the original work is properly cited, appropriate credit is given, any changes made indicated, and the use is non-commercial. See: http://creativecommons.org/licenses/by-nc/4.0/.

\section{ORCID iDs}

Tess Kramer http://orcid.org/0000-0002-2413-2329

Jan von der Thüsen http://orcid.org/0000-0001-9699-4860

\section{REFERENCES}

1 Torre LA, Bray F, Siegel RL, et al. Global cancer statistics, 2012. CA Cancer J Clin 2015;65:87-108.

2 Vilmann P, Clementsen PF, Colella S, et al. Combined endobronchial and oesophageal endosonography for the diagnosis and staging of lung cancer. European Society of gastrointestinal endoscopy (ESGE) guideline, in cooperation with the European respiratory Society (ERS) and the European Society of thoracic surgeons (ESTs). Eur Respir J 2015;46:40-60.

3 Silvestri GA, Gonzalez AV, Jantz MA, et al. Methods for staging nonsmall cell lung cancer: diagnosis and management of lung cancer, 3rd ED: American College of chest physicians evidence-based clinical practice guidelines. Chest 2013;143:e211S-50.

4 Detterbeck FC, Jantz MA, Wallace M, et al. Invasive mediastinal staging of lung cancer: ACCP evidence-based clinical practice guidelines (2nd edition). Chest 2007;132:202s-20.

5 Annema JT, van Meerbeeck JP, Rintoul RC, et al. Mediastinoscopy vs endosonography for mediastinal nodal staging of lung cancer: a randomized trial. JAMA 2010;304:2245-52.

6 Korevaar DA, Crombag LM, Cohen JF, et al. Added value of combined endobronchial and oesophageal endosonography for mediastinal nodal staging in lung cancer: a systematic review and meta-analysis. Lancet Respir Med 2016;4:960-8.

7 Kuijvenhoven JC, Leoncini F, Crombag LC, et al. Endobronchial ultrasound for the diagnosis of centrally located lung tumors: a systematic review and meta-analysis. Respiration 2020;99:441-50.

8 Skovgaard Christiansen I, Kuijvenhoven JC, Bodtger U, et al. Endoscopic ultrasound with Bronchoscope-Guided fine needle aspiration for the diagnosis of Paraesophageally located lung lesions. Respiration 2019;97:277-83.

9 Korevaar DA, Colella S, Spijker R, et al. Esophageal endosonography for the diagnosis of intrapulmonary tumors: a systematic review and meta-analysis. Respiration 2017;93:126-37.

10 Dooms C, Vliegen L, Vander Borght S, et al. Suitability of small bronchoscopic tumour specimens for lung cancer genotyping. Respiration 2014;88:371-7.

11 Kanagal-Shamanna R, Portier BP, Singh RR, et al. Next-generation sequencing-based multi-gene mutation profiling of solid tumors using fine needle aspiration samples: promises and challenges for routine clinical diagnostics. Mod Pathol 2014;27:314-27.

12 Hagemann IS, Devarakonda S, Lockwood CM, et al. Clinical nextgeneration sequencing in patients with non-small cell lung cancer. Cancer 2015;121:631-9.

13 Le Mercier M DNN, Blanchard O, et al. Clinical application of targeted next-generation sequencing for lung cancer patients. Belg J Med Oncol 2015;9:272-8.

14 Kossakowski CA, Morresi-Hauf A, Schnabel PA, et al. Preparation of cell blocks for lung cancer diagnosis and prediction: protocol and experience of a high-volume center. Respiration 2014;87:432-8.

15 Hiley CT, Le Quesne J, Santis G, et al. Challenges in molecular testing in non-small-cell lung cancer patients with advanced disease. Lancet 2016;388:1002-11.

16 Ilie M, Long-Mira E, Bence C, et al. Comparative study of the PD-L1 status between surgically resected specimens and matched biopsies of NSCLC patients reveal major discordances: a potential issue for anti-PD-L1 therapeutic strategies. Ann Oncol 2016;27:147-53.

17 Sakata KK, Midthun DE, Mullon JJ, et al. Comparison of programmed death ligand-1 immunohistochemical staining between endobronchial ultrasound transbronchial needle aspiration and resected lung cancer specimens. Chest 2018;154:827-37.

18 Bang JY, Hebert-Magee S, Navaneethan U, et al. EUS-guided fine needle biopsy of pancreatic masses can yield true histology. Gut 2018;67:2081-4.

19 Karsenti D, Palazzo L, Perrot B, et al. 22G acquire vs. 20G procore needle for endoscopic ultrasound-guided biopsy of pancreatic masses: a randomized study comparing histologic sample quantity and diagnostic accuracy. Endoscopy 2020;52:747-53.

20 Vilmann P, Clementsen PF. Combined EUS and EBUS are complementary methods in lung cancer staging: do not forget the esophagus. Endosc Int Open 2015;3:E300-1.

21 Mair S, Dunbar F, Becker PJ, et al. Fine needle cytology--is aspiration suction necessary? A study of 100 masses in various sites. Acta Cytol 1989;33:809-13.

22 van Worcum JMAD J, Radonic T. The assessment of completeness and speed in the process of diagnostic and predictive analysis of cytological EBUS-TBNA samples of lung cancer patients 2021.

23 Mineura K, Hamaji M, Yoshizawa A, et al. Diagnostic yield of endobronchial ultrasound-guided transbronchial needle aspiration to assess tumor-programmed cell death ligand-1 expression in mediastinal lymph nodes metastasized from non-small cell lung cancer. Surg Today 2020;50:1049-55.

24 Livi V, Ardizzoni A, Cancellieri A, et al. Adequacy of endosonographyderived samples from peribronchial or periesophageal intrapulmonary lesions for the molecular profiling of lung cancer. Clin Respir J 2019;13:590-7.

25 von Bartheld MB, Annema JT. Endosonography-related mortality and morbidity for pulmonary indications: a nationwide survey in the Netherlands. Gastrointest Endosc 2015;82:1009-15. 